\title{
Curvelet based Rayleigh CLAHE Medical Image Enhancement
}

\author{
Shefali Gupta \\ Research Scholar \\ Dept. of CSE \\ Chandigarh Group of Colleges Gharuan, Mohali
}

\author{
Sandeep Singh Kang, PhD \\ Professor \\ Dept. of CSE \\ Chandigarh University Gharuan, Mohali
}

\begin{abstract}
Image enhancement is one of the main issues in digital image processing. Image enhancement is done to obtain a high quality image. This makes output image better than original image. Images that are obtained from medical imaging systems are of low quality. This may happen because available range of possible gray levels may not be utilized properly. Therefore images may suffer from underexposure and overexposure problems. A new algorithm has been proposed in this paper to enhance such medical images. A comparison of existing image enhancement techniques with the proposed technique based on different performance parameters is presented. Experimental results show that proposed technique is better than various existing techniques.
\end{abstract}

\section{General Terms}

Image Enhancement

\section{Keywords}

Image enhancement, histogram equalization, adaptive histogram equalization, clahe, curvelets.

\section{INTRODUCTION}

Image enhancement is an important area in digital image processing. Some degradation may be observed while converting image from one form to another, which can be removed by enhancement. Even in the presence of advance digital technology, many real world images still appear with a low contrast. The main idea behind image enhancement is to improve the quality of the image to make the image better for some particular applications. Reducing noise, edge enhancement and contrast enhancement etc are some of the ways to enhance the image [1]. Image enhancement is one of the significant techniques in digital image processing, which plays an important role in many fields like medical image analysis, high definition television (HDTV), industrial X-ray image processing, remote sensing, microscopic imaging, satellite imaging, astronomy, real-time applications and in the other areas of research etc [2].

Various image enhancement techniques have been defined for enhancement of images. The most common technique used for image enhancement is histogram equalization (HE) [3]. In this, the gray scale histogram is uniformly distributed and the contrast is stretched among local regions thus depicting the hidden details and hence difference in the regions become more observable. Histogram Equalization has a limitation that it transforms the histogram of original image into a flat uniform histogram. Mean brightness of output image almost lies at the middle as mean value lies in the middle of gray level range after HE process. Therefore it can be said that HE method does not consider mean brightness of the input image. It also enhances the image globally, but may reduce its local content. Main disadvantage of HE process is that brightness of the image is changed after HE is applied [4].
Adaptive Histogram Equalization (AHE) is another image enhancement technique which is different from histogram equalization. This technique computes different histograms related to a particular region of an image called the contextual region and hence reconstructs the brightness values of the image using those histograms [5]. It increases local contrast but has a limitation that it over amplifies noise in homogeneous areas of the image. Also, it becomes expensive and time consuming as many histograms are to be computed for different contextual regions. Another method called Contrast Limited Adaptive Histogram Equalization (CLAHE) is an improved version of Adaptive Histogram Equalization (AHE). It restrains the problem of amplification with a clip limit criterion [6]. CLAHE has less noise, prevents brightness saturation and gives better results in local areas than other techniques like HE, AHE etc.

\section{LITERATURE SURVEY}

Kim et al. (2001) described that histogram equalization was a simple and effective image enhancement technique for enhancing the contrast of digital images. In this, pixels were assumed to be uniformly distributed over the entire image [7].

Bhadauria et al. (2011) explained curvelet based noise reduction methods. These methods were carried out on brain computed tomography (CT) images and performance assessment was calculated on the images. The experimental results showed that curvelet based methods outperformed wavelet based methods [8]

Suprijanto et al. (2012) discussed different image enhancement techniques like contrast stretching, HE, AHE, CLAHE Rayleigh. These techniques were processed on dental imaging. Contrast quality of digital image was evaluated and image quality was obtained by using CLAHE Rayleigh method of image enhancement. Objective assessment in this paper showed that CLAHE Rayleigh gave better results than other methods of enhancement. It also gave more optimal image quality [9].

Nayak et al. (2012) described the curvelet representation of the image. An analysis was made from different angles. It was shown that curvelet transform was useful in position, scale and orientation. It was also observed that the sub band concept was useful in representing objects [10].

Bedi et al. (2013) categorized image enhancement methods as spatial domain methods and frequency domain methods. In spatial domain methods direct operation on the pixels was applied. Frequency domain methods directly operate on the transform coefficients of the image like Fourier transform [11].

Min et al. (2013) explained an extended version of adaptive histogram equalization technique called contrast limited adaptive histogram equalization (CLAHE). It reduced the limitation of AHE of over amplification of noise by a clip 
limit function on the neighbourhood regions. The contrast limiting property of this technique made it better than other image enhancement techniques [12].

Jintasuttisak et al. (2014) described a method used for preserving the color information of retinal images. In this paper, the intensity component of image was enhanced by using Rayleigh CLAHE. This method increased the contrast and improved the overall appearance of the image [13].

Yadav et al. (2014) explained an image enhancement technique named contrast limited adaptive histogram equalization with Rayleigh distribution. This technique was used for the improvement of video quality in real time system [14].

Sargun et al. (2015) presented the highlights of different image enhancement techniques that could be used particularly for medical image enhancement. Limitations of some existing image enhancement methods were also discussed [15].

Anjum et al. (2015) described the use of curvelet transform for removal of noise in images. Images suffer from noise content with the process of receiving and transmission, therefore image denoising was necessary [16].

Guo et al. (2015) explained a new image enhancement method based on curvelet transform to enhance the tumor characteristic in a mammographic image. The results proved that curvelets were better than wavelets for images that had little noise, excessive noise or no noise at all [17].

Lidong et al. (2015) described the combination of CLAHE with DWT to improve the problems of contrast overstretching and noise. Experiments showed that this novel image enhancement method was better in preserving image details and suppression of noise [18].

Namdeo et al. (2016) reviewed about the distinctive image enhancement techniques like histogram equalization (HE), BBHE, adaptive histogram equalization (AHE), contrast limited adaptive histogram equalization (CLAHE) etc. with their advantages [19].

Singh et al. (2016) gave the analysis of different medical images like ultrasound images, $\mathrm{X}$ ray images etc. A study of different contrast enhancement techniques of medical images was carried out in this paper [20].

Joseph et al. (2017) made an analysis about the intensity degradation, geometric and textural features of medical images. This analysis was made in respect to clip limit variation and specified histogram shape [21].

Farzam et al. (2017) described an image contrast enhancement method for cone beam CT (CBCT) images. This was based on fast discrete curvelet changes (FDCT) that worked with unequally spaced fast Fourier transform (USFFT). The experimental results presented in this paper showed that the curvelet method worked better than wavelet method [22].

Celenk et al. (2018) explained a novel image enhancement method where CLAHE technique for image enhancement was combined with power law transformation. This method was used for ultrasonic well logging images. CLAHE technique enhanced the intensity component and excessive brightness was prevented in uniform areas. The power law transformation was applied after the CLAHE processing. The experimental results shown in this paper depicted that this technique gave better results for ultrasonic well logging images than other existing techniques [23].

\section{PROBLEM FORMULATION}

A very active research area in image processing relates to image enhancement. Some degradation may be observed whenever an image is converted from one form to another. Therefore it is processed through image enhancement. The main aim of image enhancement is to process an image so that output results are better than input image. Image enhancement is tremendously used in the field of medical diagnosis. It is observed from the past studies that due to improper results of medical imaging systems, medical images need enhancement. The results from these systems get lowered due to the presence of various hard and soft tissues or high water content in the body. The resulting image suffers from underexposure and overexposure problems. Thus, image appears grainy and of low density.

To overcome the limitations caused by the imaging systems and the limitations of existing image enhancement techniques like AHE and CLAHE, a novel image enhancement method is put forward in this paper. The goal of proposed technique is to enhance the contrast of the image while preserving the image details.

\section{PROPOSED WORK AND METHODOLOGY}

A new method, Curvelet based Rayleigh Contrast Limited Adaptive Histogram Equalization (CRCLAHE) for image enhancement of medical images is proposed. According to the proposed method, the image is first decomposed into different frequency bands (low frequency bands and high frequency bands) by curvelet transform in frequency domain. CLAHE technique with Rayleigh distribution is then applied on each of the obtained sub band for enhancement process and the enhanced image is analyzed by applying inverse curvelet transform on each frequency sub band.

The curvelet transform is a multi scale transform used as an effective tool for image enhancement. The purpose of using curvelets for image enhancement of medical images is that it has the ability to represent curves of images at different scales and directions. It can be used in positioning, scaling and orientations [9]. It can represent any discontinuity of the image more effectively than wavelets. Curvelets are processed in steps like sub band decomposition, smooth partitioning and renormalization. It is more suitable for image processing than wavelets because it can represent both smooth and edge parts of the image very well $[4,5]$.

The detailed procedure of proposed method, Curvelet based Rayleigh CLAHE (CRCAHE) is explained in the following steps:

Step1: Read the input medical image and determine its size.

Step2: Perform decomposition in the frequency domain by

decomposing input image with the curvelet transform to obtain its different frequency sub bands, low and high frequency sub bands.

Step3: On each frequency sub band, CLAHE technique with Rayleigh as a non-uniform distribution function is applied. This step will enhance the low and high frequency sub bands accordingly.

Step4: To get the output enhanced image, inverse curvelet transform is applied on the obtained sub bands.

Finally the output enhanced image generated by step 4 is visualized and saved to get the results. The results obtained 
from the proposed technique are then compared with the existing image enhancement techniques. Different image quality parameters are used to compare the results of existing techniques and proposed technique. The flow diagram for the proposed algorithm is given in fig. 1

\section{EXPERIMENTAL RESULTS}

Image enhancement on medical images and its performance evaluation is implemented using different image enhancement techniques like Histogram Equalization (HE), Adaptive Histogram Equalization (AHE), Contrast Limited Adaptive Histogram Equalization (CLAHE) with Rayleigh as a non distribution function and the proposed technique Curvelet based Rayleigh CLAHE (CRCLAHE). Here, the experiments are first carried out on CT image of head and then carried out on MRI image of head. The qualitative and quantitative results are shown below.

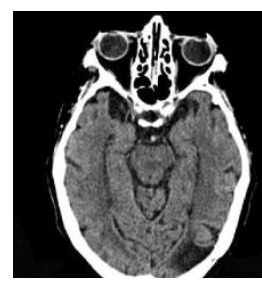

(a)

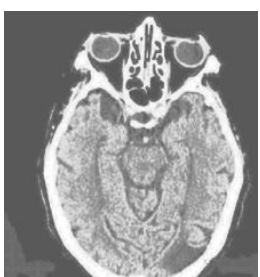

(b)

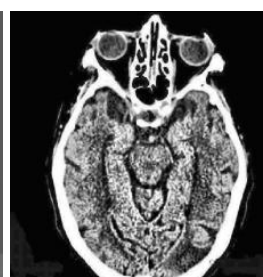

(c)

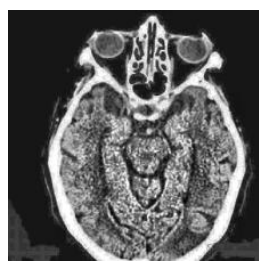

(d)

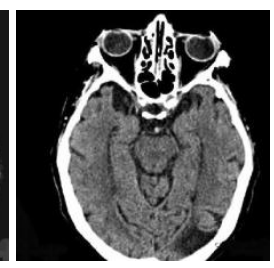

(e)
Fig 2: (a) Original CT image of head (b) HE image (c) AHE image (d) CLAHE image (e) CRCLAHE image

Figure 2 shows the qualitative analysis on CT image of head for various enhancement methods including existing and proposed method. Image quality parameters like Peak-Signalto-Noise Ratio (PSNR), Normalized Absolute Error (NAE), Maximum Difference (MD) and Structural Content (SC) are calculated on the images to show the quantitative results.

Table 1: Comparison of image enhancement techniques on the basis of image quality parameters for CT image of head

\begin{tabular}{|l|l|l|l|l|}
\hline $\begin{array}{l}\text { Technique/ } \\
\text { Parameter }\end{array}$ & PSNR & NAE & MD & SC \\
\hline HE & 10.082 & 1.0861 & 16 & 0.48208 \\
\hline AHE & 19.309 & 0.25538 & 56 & 0.78898 \\
\hline RCLAHE & 18.4863 & 0.3684 & 68 & 0.88564 \\
\hline CRCLAHE & $\mathbf{4 8 . 3 1 7 9}$ & $\mathbf{0 . 0 0 8 2 2 4 1}$ & $\mathbf{5}$ & $\mathbf{1 . 0 0 0 7}$ \\
\hline
\end{tabular}

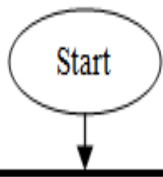

Frequency Domain decomposition of input image with curvelet transform to obtain frequency sub-bands

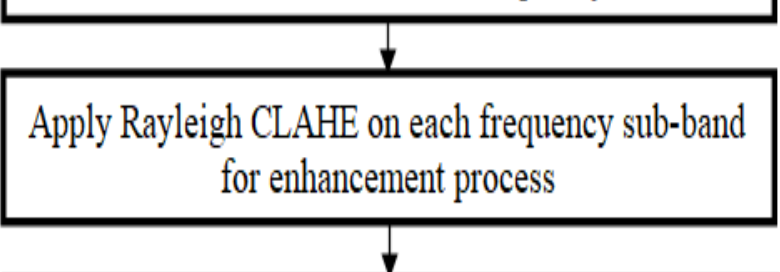

Apply inverse curvelet transform on obtained frequency sub-bands to get the enhanced image

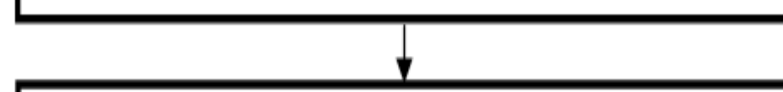

Compare the results with existing techniques to show proposed technique gives better results

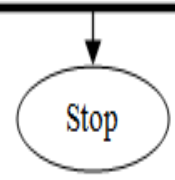

Fig 1: Flow diagram of proposed method

It is observed from Table 1 that values of these parameters for proposed technique are better than other existing image enhancement techniques. Here, higher value of PSNR and SC in case of proposed method makes it better in comparison to other existing techniques.

Figure 3 shows the analysis of existing histogram equalization based image enhancement techniques and the proposed technique for MRI image of head. The processed images obtained after applying existing image enhancement techniques are compared with the original image.

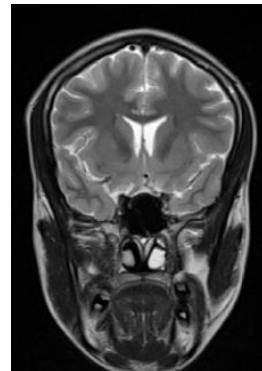

(a)

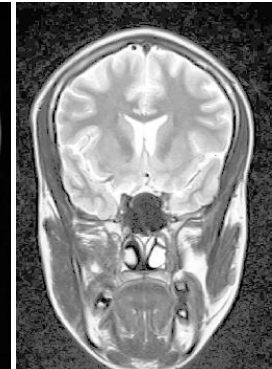

(b)

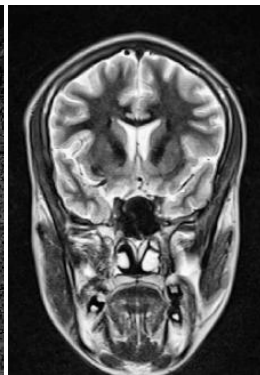

(c) 


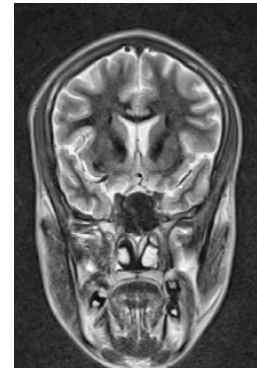

(d)

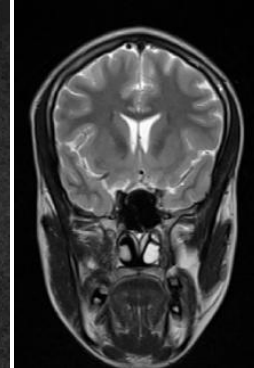

(e)
Fig 3: (a) Original MRI image of head (b) HE image

(c) AHE image (d) CLAHE image (e) CRCLAHE image

The following table 2 represents the summary of results for existing techniques and the proposed technique on input medical MRI image of head. The values of PSNR, NAE, MD and SC makes proposed algorithm better than other existing techniques.

Table 2: Comparison of image enhancement techniques on the basis of image quality parameters for MRI image of head

\begin{tabular}{|l|l|l|l|l|}
\hline $\begin{array}{l}\text { Technique/ } \\
\text { Parameter }\end{array}$ & PSNR & NAE & MD & SC \\
\hline HE & 10.1716 & 1.3587 & 0 & 0.27588 \\
\hline AHE & 16.7128 & 0.52949 & 53 & 0.51817 \\
\hline RCLAHE & 15.6366 & 0.70301 & 52 & 0.53038 \\
\hline CRCLAHE & $\mathbf{4 8 . 4 4 8 2}$ & $\mathbf{0 . 0 1 1 0 4 6}$ & $\mathbf{5}$ & $\mathbf{1 . 0 0 0 2}$ \\
\hline
\end{tabular}

Figures 4, 5, 6 and 7 in the following part represents the graphical comparison of existing image enhancement techniques with the proposed technique based on different image quality parameters, named, PSNR, NAE, MD and SC for different medical images taken in this work.

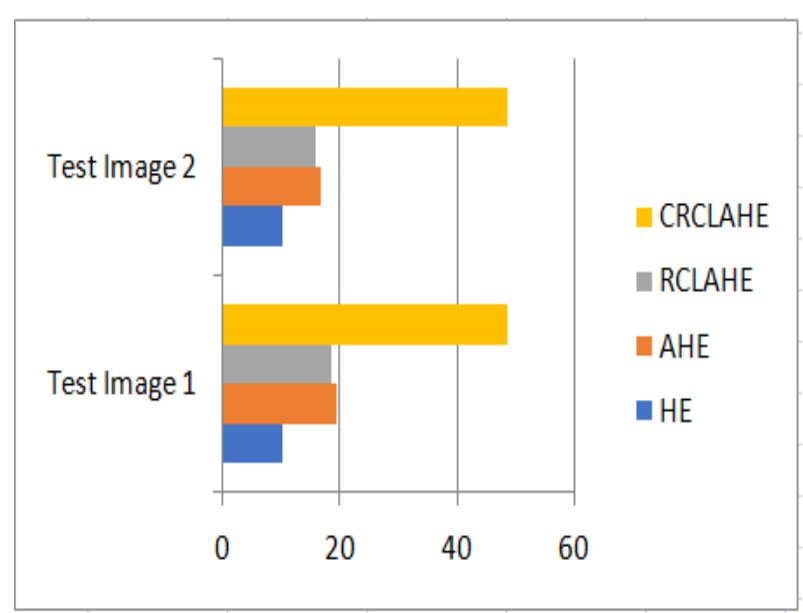

Fig.4: Analysis of PSNR for existing and proposed methods

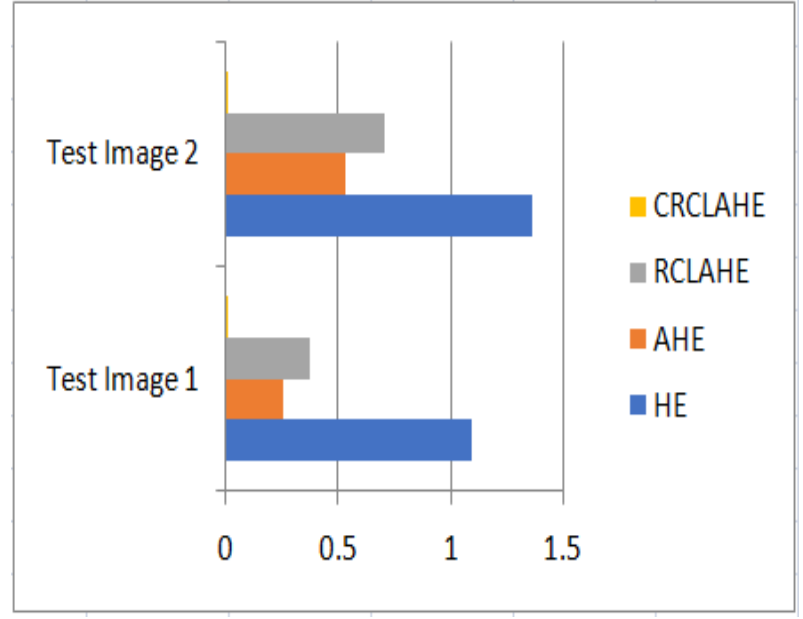

Fig.5: Analysis of NAE for existing and proposed methods

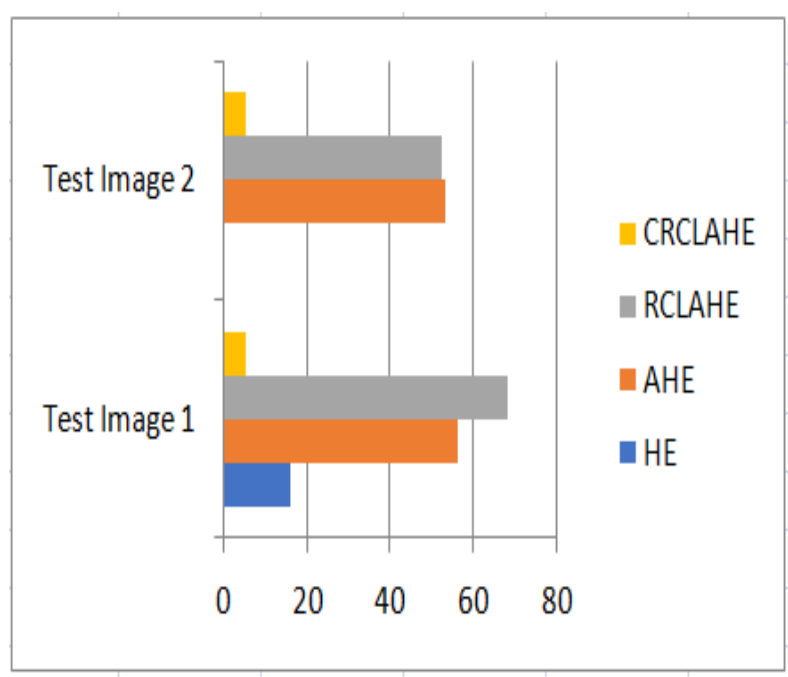

Fig.6: Analysis of MD for existing and proposed methods

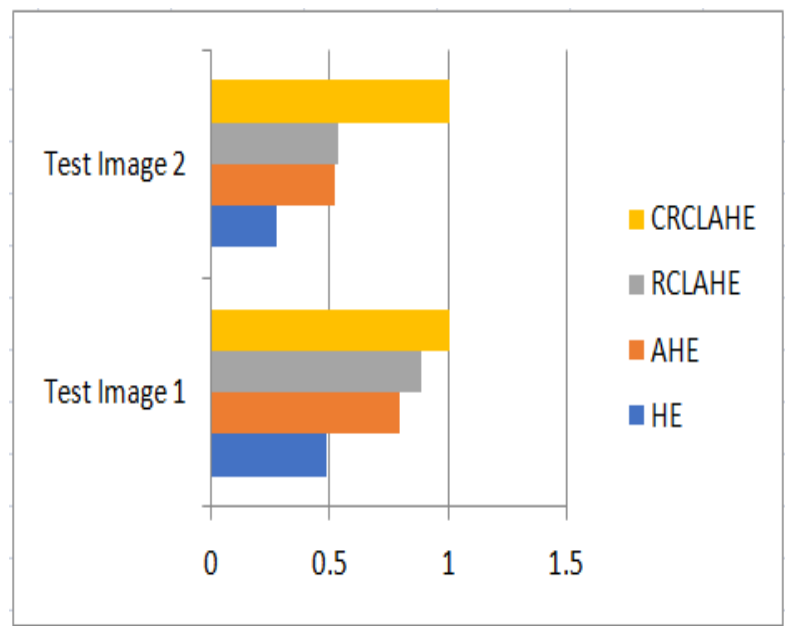

Fig.7: Analysis of SC for existing and proposed methods

6. CONCLUSION AND FUTURE SCOPE

In digital image processing, image enhancement is one of the important areas. One of the main existing image enhancement techniques is Histogram equalization (HE). In this paper, the existing techniques like HE, AHE and CLAHE are implemented for enhancement of medical images. A new method for image enhancement of medical images has been 
proposed. It enhances the contrast of images and preserves the brightness. The proposed technique is implemented using MATLAB. The evaluation is based on image quality parameters like peak-signal-to-noise ratio (PSNR), Normalized Absolute Error (NAE), Maximum Difference (MD) and Structural Content (SC). It is observed that the proposed method gives higher values of PSNR and SC and thus depicts more good quality image. Other quantitative results of NAE and MD also show that proposed method gives more optical quality of image. It improves the overall appearance of image so that the observer can extract the required information. In future, the performance of proposed method can be investigated at a larger scale to other medical images like ultrasound images and X-ray images etc. Some more image quality parameters can be calculated to make a much better comparison in the results. The proposed method can be tested for medical video which is yet another challenging area.

\section{REFERENCES}

[1] Gonzalez, R. C., \& Woods, R. E. 2013. Digital Image Processing. NJ: Prentice Hall of India.

[2] Singh, S., Bansal, R. K., \& Bansal, S. 2012. Comparative Study and Implementation of Image Processing Techniques Using MATLAB. International Journal of Advanced Research in Computer Science and Software Engineering, 2(3)

[3] Stark, J. A. 2000. Adaptive Image Contrast Enhancement Using Generalizations of Histogram Equalization. IEEE Transactions on Image Processing, 9(5), 889-896.

Gupta, S., Kang, S. S. 2018. Image Enhancement of Medical Images using Curvelet and Rayleigh CLAHE. IOSR Journal of Computer Engineering, IOSR-JCE, 20(3), 6980.

Pizer, S. M., Amburn, E. P., Austin, J. D., Cromartie, R., Geselowitz, A., Greer, T., Romeny, B. H., Zimmerman, J. B., \& Zuiderveld, K. 1987. Adaptive Histogram Equalization and Its Variations. Computer Vision Graphics and Image Processing, 39, 355-368.

[4] Pujiono, Pulung, N.A., Purnama, I. K. E., \& Hariadi, M. 2013. Color Enhancement of Underwater Coral Reef Images using Contrast Limited Adaptive Histogram Equalization with Rayleigh Distribution. The Proceedings of The 7th ICTS.

[5] Kim, J. Y., Kim, L. S., \& Hwang, S. H. 2001. An Advanced Contrast Enhancement using Partially Overlapped Sub-Block Histogram Equalization. IEEE Transactions on Circuits and Systems for Video Technology, 11(4), 475-484.

[6] Bhadauria, H. S., Dewal, M. L., \& Anand, R. S. 2011. Comparative Analysis of Curvelet based techniques for denoising of Computed Tomography Images. IEEE.

[7] Suprijanto, G., Juliastuti, E., Azhari, \& Epsilawati, L. 2012. Image Contrast Enhancement for Film-Based Dental Panoramic Radiography. International Conference on System Engineering and Technology, IEEE International Conference on.
[8] Nayak, R., Bhavsar, J., Chaudhari, J., \& Mitra, S. K. 2012. Object tracking in Curvelet Domain with dominant Curvelet Subbands. International Journal of Engineering Research and Applications (IJERA), 2(3), 1219-1225.

[9] Bedi, S. S., \& Khandelwal, R. 2013. Various Image Enhancement Techniques- A Critical Review. IJARCCE, 2(3), 1605-1609.

[10] Min, B. S., Lim, D. K., Kim, S. J., \& Lee, J. H. 2013. A Novel Method of Determining Parameters of CLAHE Based on Image Entropy. International Journal of Software Engineering and Its Applications, 7(5), 113120

[11] Jintasuttisak, T., \& Intajag, S. 2014. Color Retinal Image Enhancement by Rayleigh Contrast-Limited Adaptive Histogram Equalization. $14^{\text {th }}$ International Conference on Control, Automation and Systems, ICROS, 692-697.

[12] Yadav, G., Maheshwari, S., \& Agarwal, A. 2014 Contrast Limited Adaptive Histogram Equalization Based Enhancement For Real Time Video System. International Conference on Advances in Computing, Communications and Informatics, ICACCI, IEEE, 23922397.

[13] Sargun, \& Rana, S. B. 2015. A Review of Medical Image Enhancement Techniques for Image Processing. International Journal of Current Engineering and Technology, 5(2), 1282-1286.

[14] Anjum, K., \& Bhyri, C. 2015. Image Denoising Using Curvelet Transform and Edge Detection in Image Processing, Proceeding of NCRIET.

[15] Guo, Q., \& Su, X. 2015. The study of medical image enhancement based on curvelet. Technology and Health Care 23, S319-S323.

[16] Lidong, H., Wei, Z., Jun, W., \& Zebin, S. 2015 Combination of contrast limited adaptive histogram equalization and discrete wavelet transform for image enhancement. IET Image Processing, 9(10), 908-915.

[17] Namdeo, A., \& Bhadoriya, S. S. 2016. A Review on Image Enhancement Techniques with its Advantages and Disadvantages. IJSART, 2(5), 171-182.

[18] Singh, S., \& Pal, P. 2016. Contrast Enhancement of Medical Images: A Review. IJRDO-Journal of Health Sciences and Nursing, 1(4), 32-35.

[19] Joseph, J., Sivaraman, J., Periyasamy, R., \& Simi,V. R 2017. An objective method to identify optimum cliplimit and histogram specification of contrast limited adaptive histogram equalization for MR images. Biocybernetics and Biomedical Engineering, Science Direct, Elsevier, 489 - 497.

[20] Farzam, S., \& Rastgarpour, M. 2017. An Image Enhancement Method Based on Curvelet Transform for CBCT Images. International Journal of Computer and Information Engineering, 11(6), 215-221.

[21] Fu, Q., Celenk, M., \& Wu, A. 2018. An improved algorithm based on CLAHE for ultrasonic well logging image enhancement. Cluster Computing, Springer. 